\title{
Retrospective Study of Non-Responding Patients to ATT Presenting To the RNTCP Center of a Tertiary Care Hospital
}

\author{
Authors \\ Dr Naveen kumar Verma ${ }^{1}$, Dr Raghvendra Singh Gaur $^{2}$, Dr Rajni Prajapati ${ }^{3}$ \\ Dr Swati Chandel ${ }^{4}$, Dr Karan Singh Chandrakar ${ }^{5}$ \\ ${ }^{1}$ Assistant Professor Department of Pathology, CCM Medical College Durg C.G \\ ${ }^{2}$ Assistant Professor, Department of ENT, CCM Medical College Durg C.G, \\ ${ }^{3}$ Demonstrator, Department of Pathology, CCM Medical College Durg C.G, \\ ${ }^{4}$ Senior Resident, Department of ENT, CCM Medical College Durg C.G, \\ ${ }^{5}$ Assistant Professor Department of Pathology, CCM Medical College Durg C.G \\ RNTCP center of R.D. Gardi Medical College, Ujjain, MP \\ Correspondent Author \\ Dr Naveen Kumar Verma \\ Department of Pathology, CCM Medical College, Durg, CG \\ Mobile number - 9630450461
}

\begin{abstract}
Tuberculosis $(T B)$ is a pandemic and half of the cases are in six Asian countries. WHO estimates that at least one third of the world population is infected with Mycobacterium tuberculosis. It is a major health issue; its treatment is more expensive of a longer duration and gives less than ideal cure rates with higher relapse rates. In this study all patients who were not responding to medical treatment of ATT were assessed. A total of 74 case sheets from the outdoor patient clinic selected, of which $73 \%$ (54 cases) of cases were male and $27 \%$ (20 cases) cases were female. Data collected in heading of Smear, culture and sensitivity and PCR reaction. $86 \%$ (64 cases) cases were sputum positive, out of which $76.6 \%$ (49 cases) were male and $23.4 \%$ (15 cases) female. $72.9 \%$ (54 cases) sample was positive on culture, out of which male were $76 \%$ (41 cases) and female $24 \%$ (13 cases). All sample were positive for PCR, out of which $75.7 \%$ (56 cases) were male and $24.3 \%$ (18 cases) were female. Segregation of data done according to category. Immunocompromised status was taken in account and only 1 patient found HIV positive and was a case of pulmonary tuberculosis. At present, it can be concluded that the smear is cheap and rapid method of detecting mycobacterium tuberculosis but it has a very low sensitivity. Culture is more sensitive but it takes a longer time to give results while PCR is specific, rapid, more sensitive but expensive technique, and it should only be used in difficult cases where diagnosis become a challenge.
\end{abstract}


Aims -:

$>$ To find out pattern of non-responding patients presenting to the tertiary care RNTCP center.

$>$ Distribution of MDR resistance cases according to age and gender.

$>$ Their resistance to anti tubercular medicine.

$>$ Distribution of MDR cases in relation to Category.

$>$ Immuno-compromised status of patient.

\section{Inclusion criteria}

- Patients not responding to medical management (ATT)

\section{Exclusion criteria}

- Patient responding to ATT.

\section{Introduction}

Tuberculosis (TB) is a pandemic and half of the cases are in six Asian countries (Bangladesh, China, India, Indonesia, Pakistan and the Philippines).WHO estimates that at least one third of the world population is infected with Mycobacterium tuberculosis. It is a major health issue; its treatment is more expensive of a longer duration and gives less than ideal cure rates with higher relapse rates. In this study all patients who were not responding to medical treatment of ATT were assessed.

The term "co-epidemic" or "dual epidemic" was applied for HIV/AIDS and TB presented simultaneously. The incidence of MDR-TB is astonishingly high in HIV/AIDS patients. Patients who are HIV positive have an increased bacterial load due to poor T-cell immunity, higher the bacterial load more the number of drug resistant and there is a problem of drug absorption in these patients due to chronic diarrhea.

Microscopic examination of Zheil Neelsen stained smears for acid-fast bacilli and culture on Lowenstein Jensen (LJ) media are the methods of choice for the diagnosis of Mycobacterium tuberculosis in most developing countries. Conventional diagnosis of Pulmonary
Tuberculosis (PTB) is time-consuming and the acid fast bacilli (AFB) smear (Zheil Neelsen staining) is a cheap and specific test which takes about 1 to 2 hours for reporting, however it is less sensitive $(40 \%-60 \%)^{1}$ and requires a large number of bacilli (up to 10,000 bacilli/ml) in the specimen. Moreover, it can not distinguish Mycobacterium tuberculosis from Mycobacterium other than tuberculosis and is therefore, used for screening only ${ }^{2}$. This technique is widely used in India and other developing countries ${ }^{3}$.

Culture is the traditional method of confirming the diagnosis of tuberculosis. However, because the organisms are slowly growing, laboratory diagnosis by conventional methods can take as long as 10 weeks. Despite the acceptance of culture as the definitive tool for the diagnosis of tuberculosis, some microscopy-positive specimens fail to yield mycobacteria on culture. This may be due to the harsh chemical treatment which is used to decontaminate specimens, to contamination with other bacteria, or to the presence of nonviable mycobacteria. A recent report by Daniel $^{4}$ estimates that the sensitivity of culture can be as low as $50 \%$. Serodiagnosis using different antigens does not have sufficient sensitivity and specificity and tuberculin test used for screening does not indicate active infection ${ }^{5}$. Polymerase chain reaction (PCR) test for the diagnosis of tuberculosis is not well evaluated in developing countries $^{6}$ but it has already begun to effect clinical investigation ${ }^{7}$. This study was done to compare the PCR for the rapid diagnosis of Mycobacterium tuberculosis for both pulmonary and extra-pulmonary specimens, and compare the results with acid-fast bacilli smear and culture.

\section{Material and method}

Data of 74 patients were collected from the outpatient clinic. According to data all samples were obtained from patients who were not responding for ATT. 


\section{Method for Acid-fast staining:}

A direct smear made from each sputum specimen and stained by the Zheil Neelsen (ZN) method. Processing of samples - an equal volume of $\mathrm{N}$ acetyl-L-cysteine- $\mathrm{NaOH}$ solution added into the sputum sample, and the content shaken for 20-30 minutes and allowed to stand for 10 minutes. The digested/decontaminated sputum sample then diluted with sterile phosphate buffered saline $(\mathrm{pH}$ 6.8) and centrifuged at 4,000 rpm for 20 minutes at room temperature. The pellet was then used to

1. Prepare smear on a glass slide for $\mathrm{ZN}$ staining.

2. Resuspended in $500 \mathrm{~mL}$ of phosphate buffered saline to inoculate LJ medium.

3. Processed for PCR detection of TB.

Direct and concentrated ZN smears prepared from the specimens. A minimum of 100 oil fields observed before declaring a smear as negative or positive. The results reported using the criteria laid down by $\mathrm{WHO} /$ International Union of against
Tuberculosis and Lung Diseases (IUATLD). When any red bacilli are seen, report the smear as AFB positive. More then 10 AFB per field at least in twenty field report as +++, 1-10 AFB per field at least in 50 field report as,$++ 10-99$ AFB per 100 field report as +, 1-9 AFB per 100 field report the exact number.

\section{PCR method for detection of TB}

The following reaction cycles used with use of primers: an initial 4-minute denaturation step at $94 * \mathrm{C}$ followed by 35 cycles of amplification $\left(94 * \mathrm{C}\right.$ for 45 seconds, $68^{*} \mathrm{C}$ for 45 seconds, and $72 * \mathrm{C}$ for 1 minute). The $72 * \mathrm{C}$ extension step extended for an additional 5 minutes. The reaction stopped by cooling at $4 * \mathrm{C}$, and the PCR amplified products analyzed by $2.5 \%$ agarose gel electrophoresis, and $\mathrm{M}$ tuberculosis complex IS6110- specific DNA band corresponding to 123 bp detected by Gel Doc 1000 Trans-illuminator Positive and negative controls run with each batch or sample analyzed.

\section{Observation}

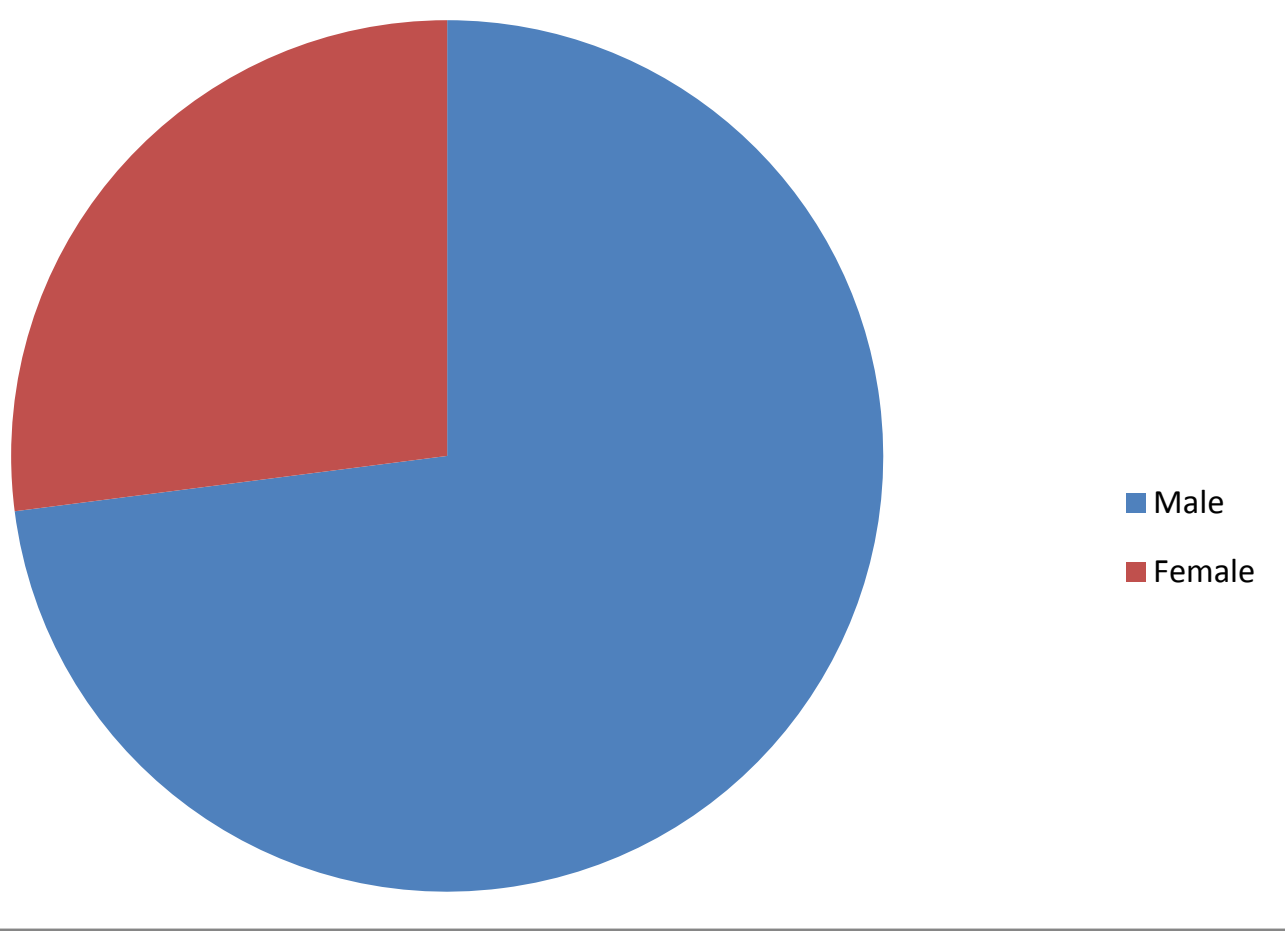

Table 1. Distribution of patients according to gender 


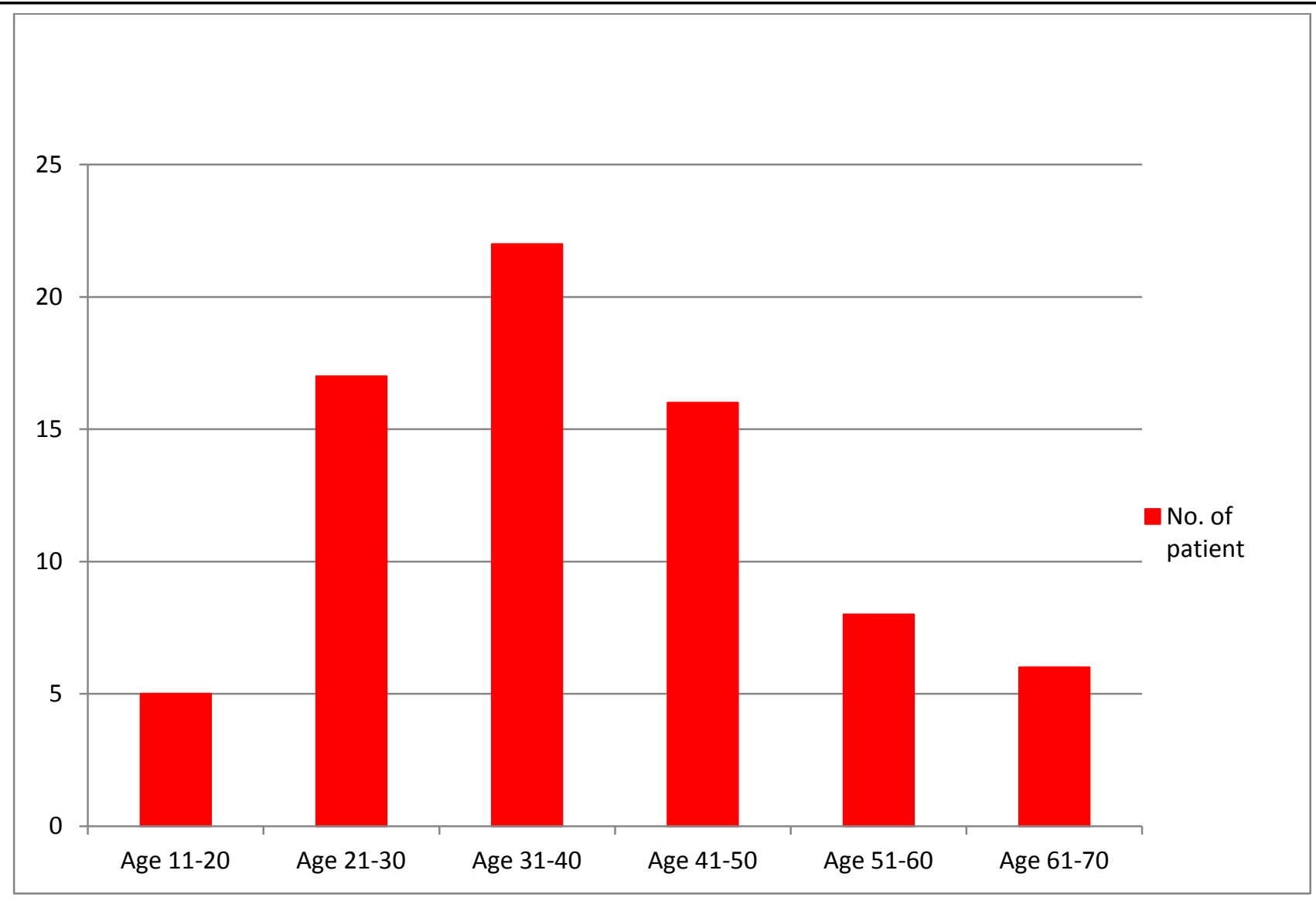

Table 2. Distribution of patients according to age

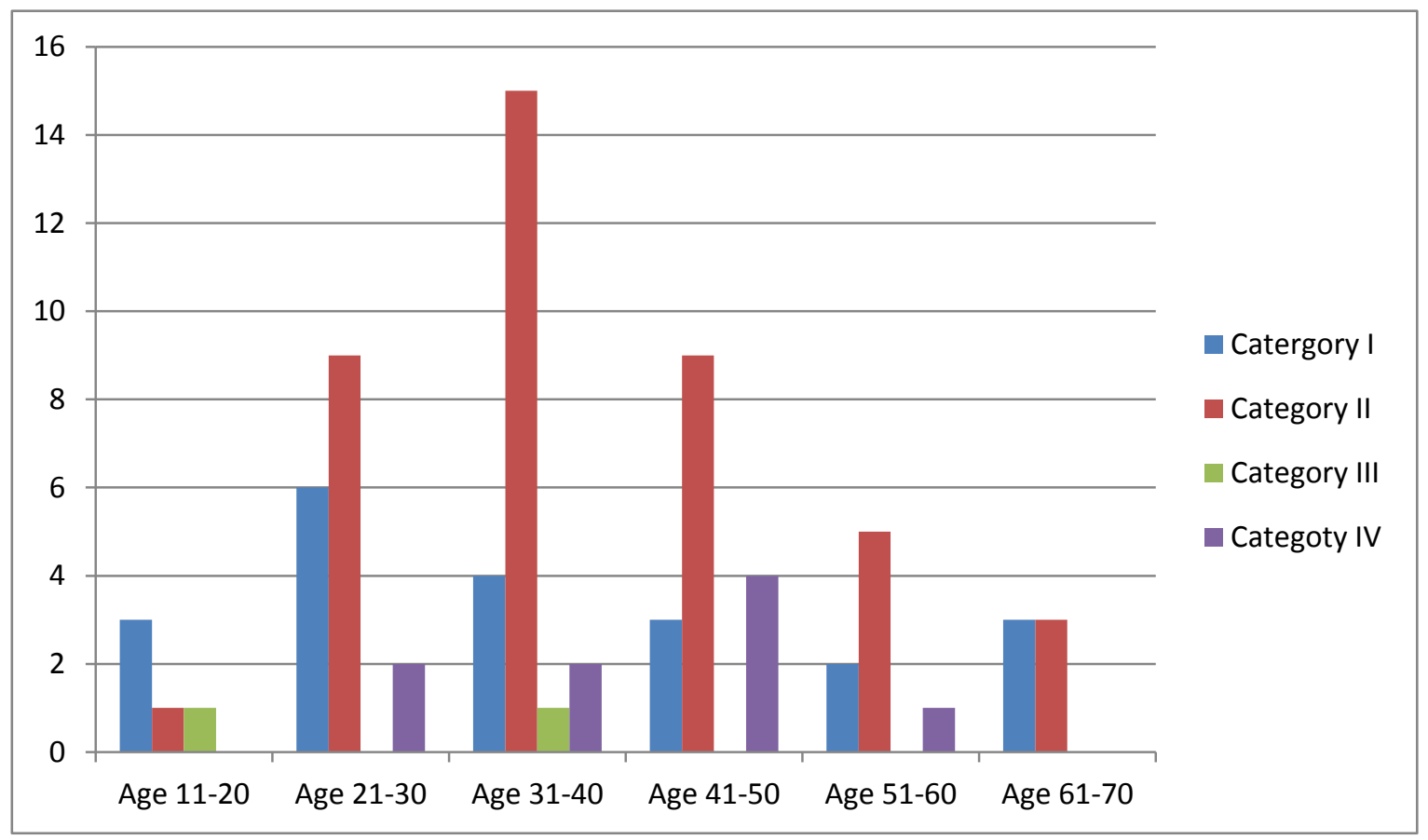

Table 3. Distribution of patients according to category 


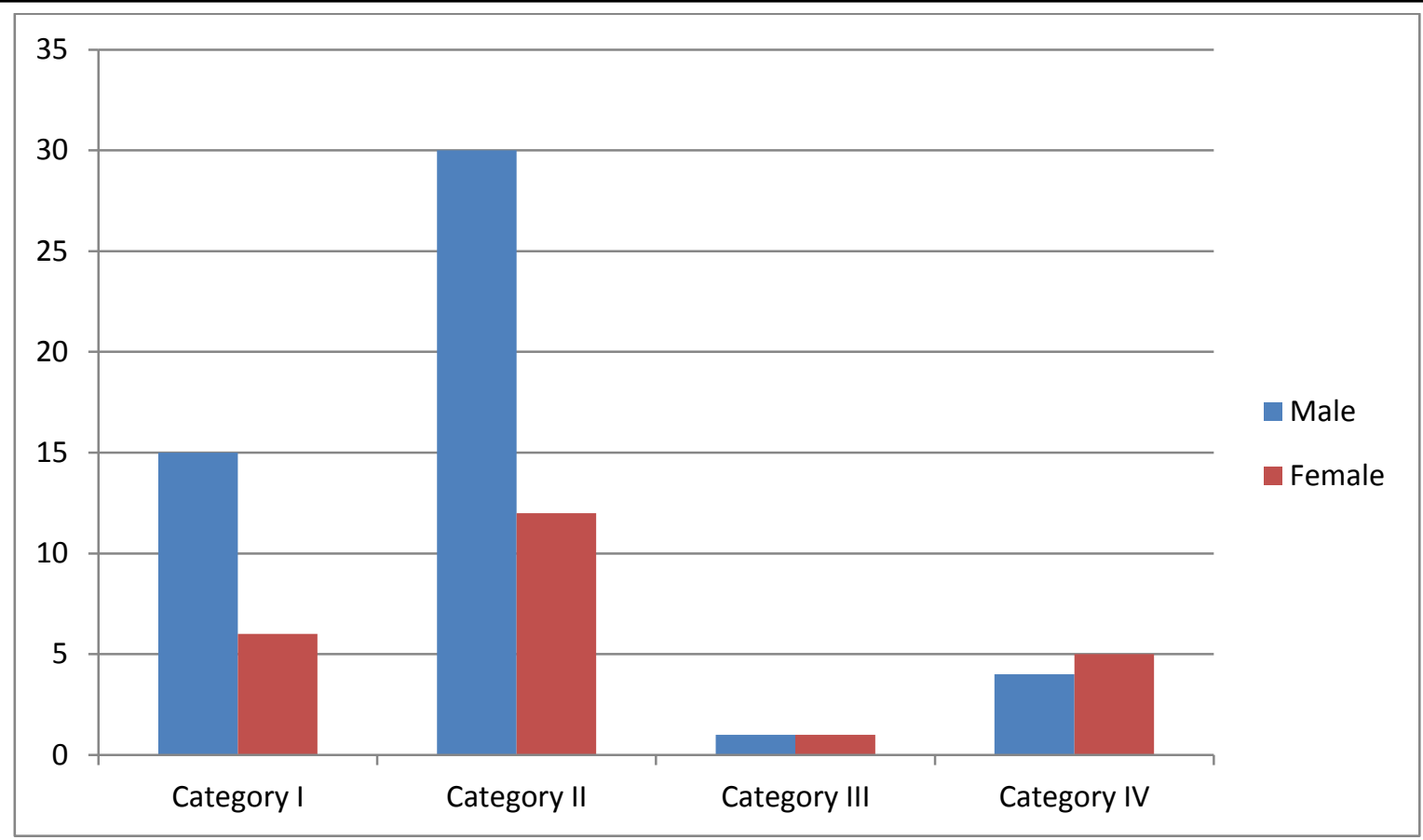

Table 4. Distribution of patients gender according to category

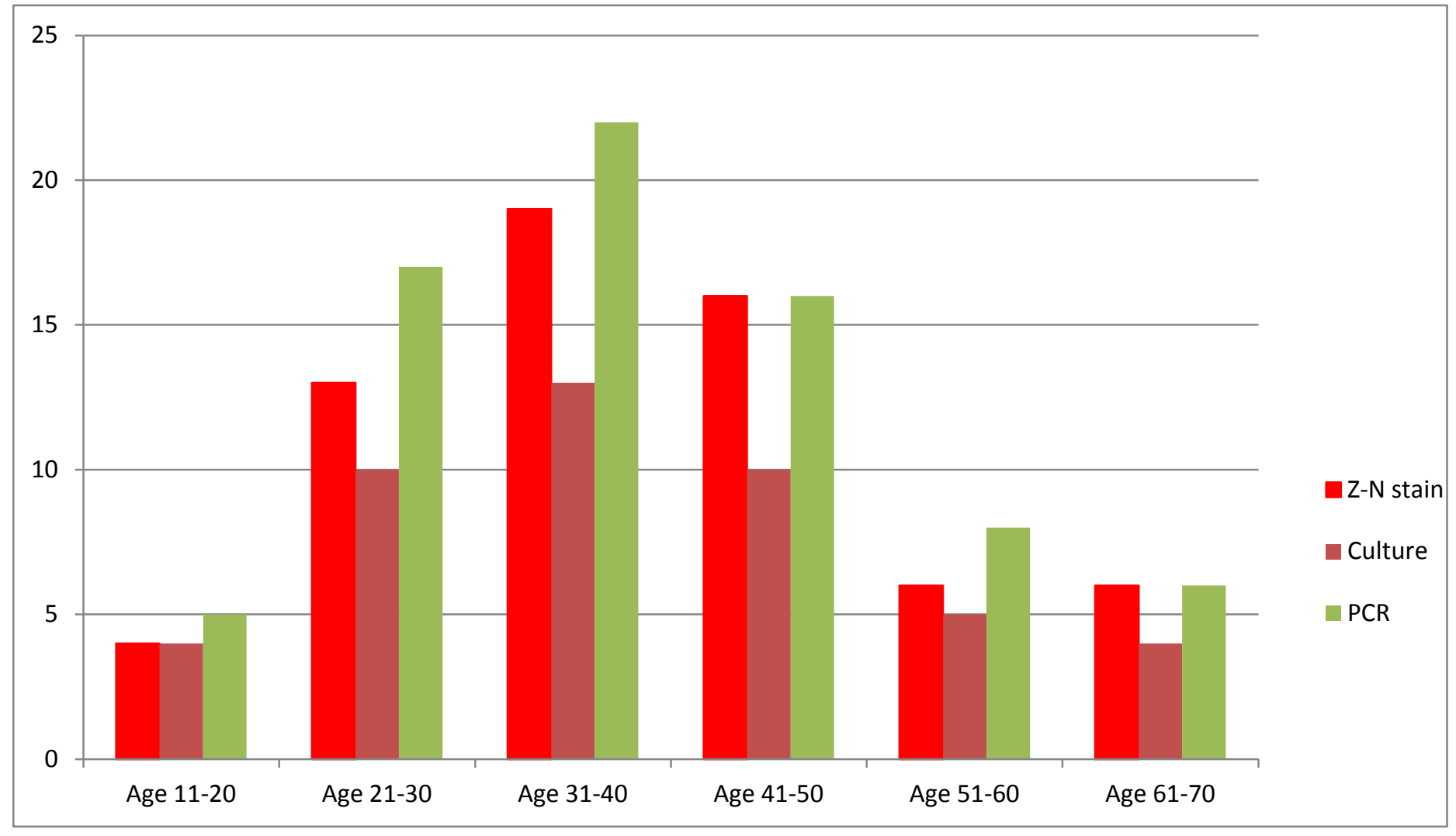

Table 5. Distribution of $\mathrm{ZN}$ stain, Culture and PCR result according to age 


\section{JMSCR Vol||4||Issue||02||Page 9187-9196||February}

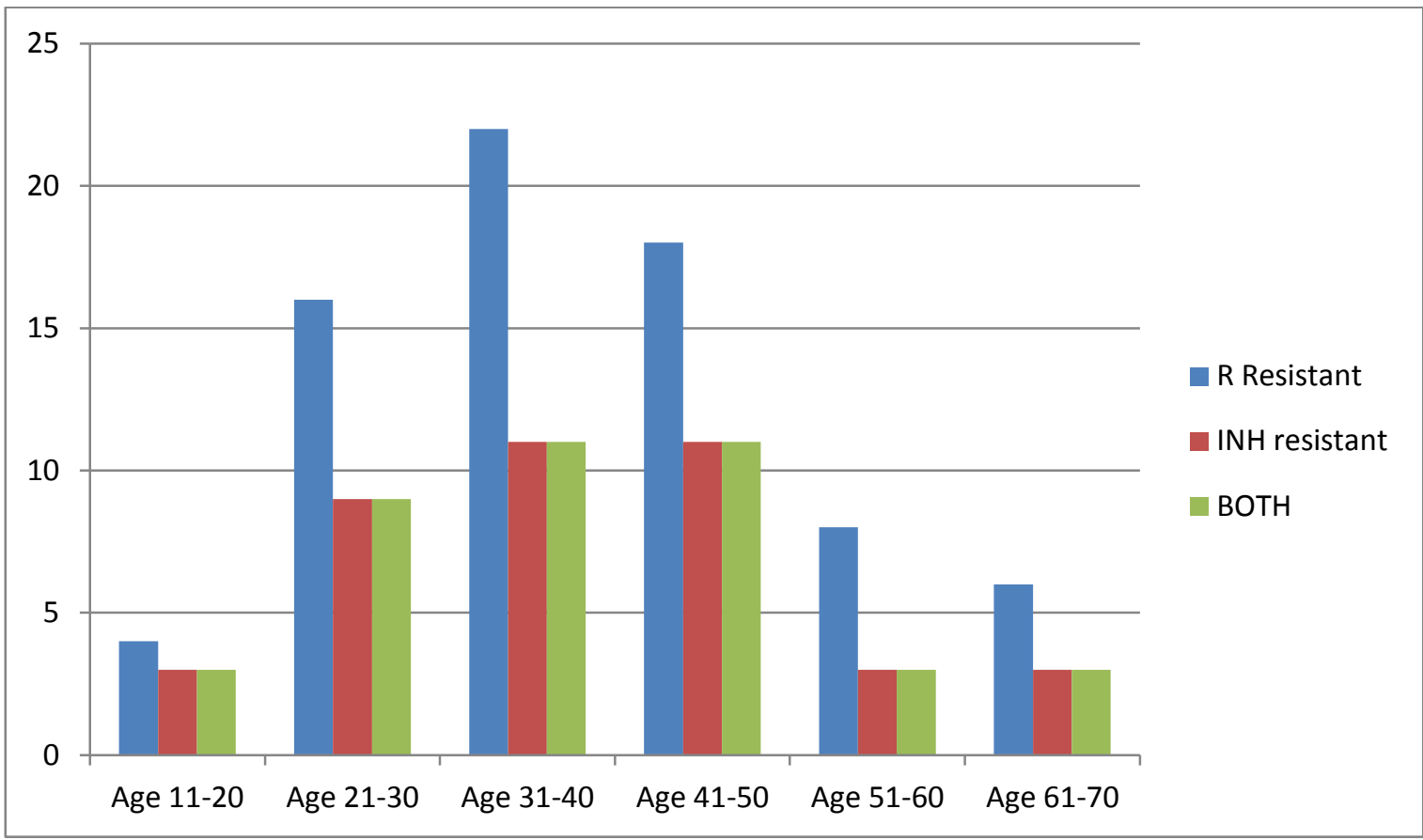

Table 6. Distribution of patients according to Resistant to R and INH

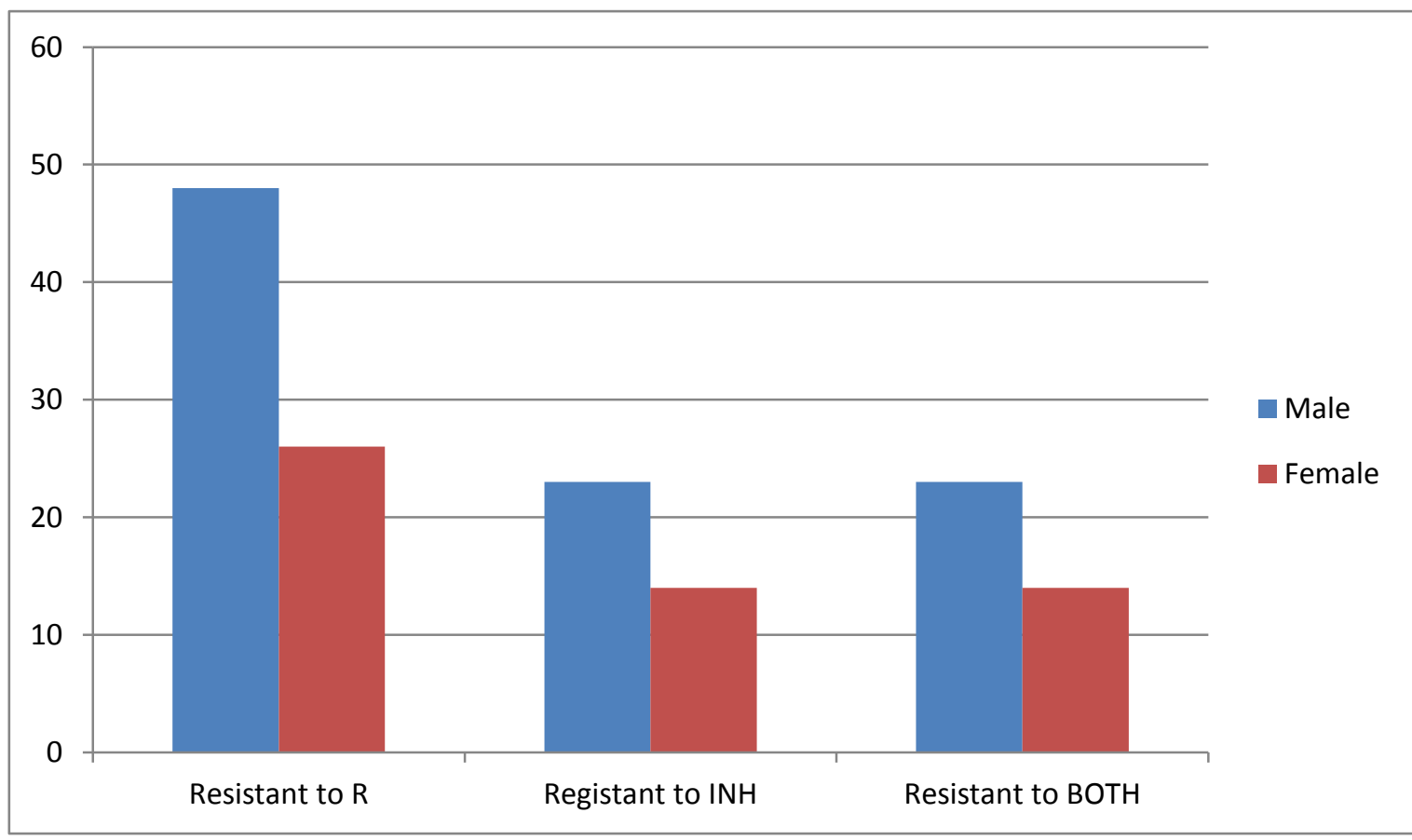

Table 7. Distribution of patients gender according to Resistant to R and INH 


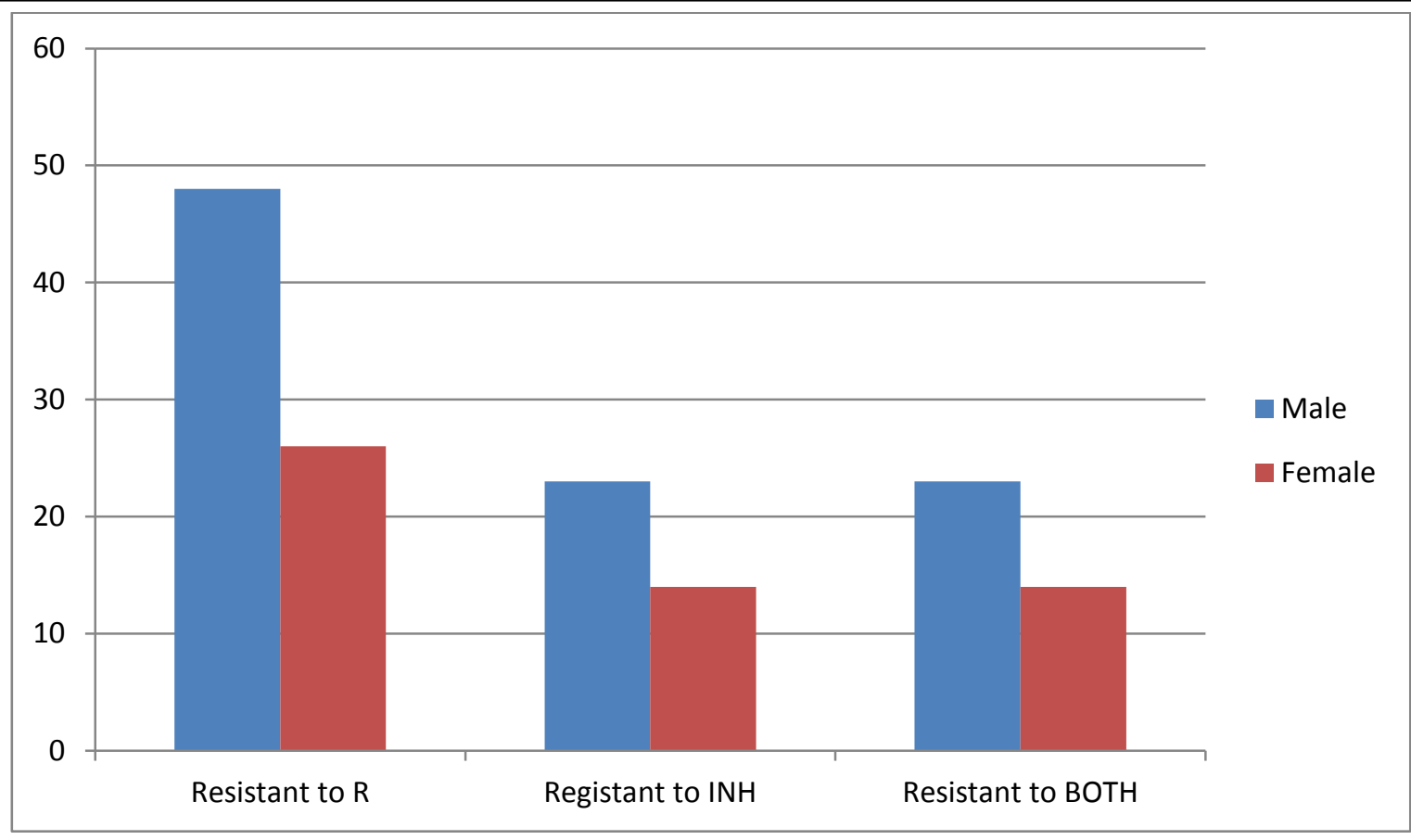

Table 8. Distribution of patients gender according to resistant to R ang INH

\section{Result}

In this study patients referred to the MDR center of the tertiary care hospital were included. Total numbers of patients were 74 , out of which $73 \%$ (54 cases) of cases were male and $27 \%$ (20 cases) cases were female. Age of the youngest patient was $16 \mathrm{yrs}$ and oldest patient was $70 \mathrm{yrs.} 86 \%$ (64 cases) cases were sputum positive, out of which $76.6 \%$ (49 cases) were male and $23.4 \%$ (15 cases) female. Sputum smear data segregation done according to the age groups showed, age group of 11-20 had $6.2 \%$ (4 cases) cases out of which $1.5 \%$ ( 1 case) male and $4.6 \%$ ( 3 cases) female, $21-30$ age group $20.3 \%$ (13 cases) out of which $15.6 \%$ (10 cases) male and $4.6 \%$ (3 cases) female, 31-40 age group 29.6\% (19 cases) male $25 \%$ (16 cases) female $4.6 \%$ (3 cases), 41- 50 age group $25 \%$ (16 cases) out of which male 18.8 $\%$ (12 cases) and $6.3 \%$ ( 4 cases) female, 51- 60 age group $9.3 \%$ ( 6 cases) out of which male 9.3 $\%$ (6 cases) and no female found, 61 -70 age group $9.3 \%$ (6 cases) out of which male $6.3 \%$ ( 4 cases) and female $3.1 \%$ ( 2 cases).

$72.9 \%$ (54 cases) sample was positive on culture, out of which male were $76 \%$ (41 cases) and female $24 \%$ (13 cases). Culture report data segregation done according to the age groups showed, age group of 11-20 had 7.4 \% (4 cases) cases out of which $1.9 \%$ (1 case) male and $5.6 \%$ ( 3 cases) female, 21- 30 age group $20 \%$ (11 cases) out of which $13 \%$ (7 cases) male and 7.4 $\%$ (4 cases) female, 31-40 age group 31.5\% (17 cases) male $27.8 \%$ (15 cases) female $3.7 \%$ (2 cases), 41- 50 age group $22.2 \%$ (12 cases) out of which male $16.7 \%$ (9 cases) and $5.6 \%$ ( 3 cases) female, 51- 60 age group $9.3 \%$ ( 5 cases) out of which male $9.3 \%$ (5 cases) and no female found, $61-70$ age group $9.3 \%$ (5 cases) out of which male $7.4 \%$ ( 4 cases) and female $1.9 \%$ (1case).

All samples were positive for PCR, out of which $75.7 \%$ (56 cases) were male and $24.3 \%$ (18 cases) were female. PCR report data segregation done according to the age groups showed, age group of 11-20 had $6.7 \%$ (5 cases) cases out of which $2.7 \%$ (2 case) male and $4 \%$ ( 3 cases) female, 21 - 30 age group $23 \%$ (17 cases) out of which $17.6 \%$ (13 cases) male and $5.4 \%$ (4 cases) female, 31-40 age group 30\% (22 cases) male $24.3 \%$ (18 cases) female $5.4 \%$ (4cases), $41-50$ age group $21.6 \%$ (16 cases) out of which male $16.2 \%$ (12 cases) and $5.4 \%$ ( 4 cases) female, 5160 age group $10.8 \%$ ( 8 cases) out of which male $9.4 \%$ (7 cases) and $1.3 \%$ (1 case) female found, 
$61-70$ age group $8 \%$ (6 cases) out of which male $5.4 \%$ ( 4 cases) and female $2.7 \%$ ( 2 cases).

Segregation of data done according to category. In category I age group of 11-20 had $4 \%$ (3 cases) cases out of which $1.3 \%$ (1 case) male and $2.7 \%$ ( 2 cases) female, $21-30$ age group $8 \%$ (6 cases) out of which $6.7 \%$ (5 cases) male and $1.3 \%$ (1 case) female, 31-40 age group $5.3 \%$ (4 cases) male $4 \%$ ( 3 cases) female $1.3 \%$ ( 1 case), $41-50$ age group $4 \%$ (3 cases) out of which male $4 \%$ (3 cases) and none female, 51- 60 age group $2.6 \%$ ( 2 cases) out of which male $1.3 \%$ ( 1 case) and 1.3 $\%$ (1 case) female found, $61-70$ age group $4 \%$ (3 cases) out of which male $2.7 \%$ ( 2 cases) and female $1.3 \%$ (1case).

Segregation of data done according to category. In category II age group of 11-20 had $1.3 \%$ (1 case) cases out of which none male and $1.3 \%$ ( 1 case) female, 21 - 30 age group $11.1 \%$ (9 cases) out of which $10.8 \%$ (8 cases) male and $1.3 \%$ (1 case) female, 31-40 age group $20.2 \%$ (15 cases) male $13.5 \%$ (10 cases) female $6.7 \%$ (5 cases), 41- 50 age group $12.1 \%$ (9 cases) out of which male 6.7 $\%$ (5 cases) and $5.4 \%$ (4 cases) female, 51- 60 age group $6.7 \%$ ( 5 cases) out of which male 6.7 $\%$ (5 cases) and none female found, 61 -70 age group $4 \%$ (3 cases) out of which male $2.7 \%$ ( 2 cases) and female $1.3 \%$ (1 case).

Segregation of data done according to category. In category III age group of 11-20 had $1.3 \%$ (1 case) cases out of which none male and $1.3 \%$ ( 1 cases) female, 21- 30 age group, no case found, 31-40 age group $1.3 \%$ (1 case) male $1.3 \%$ (1 case) none female, no case found in the age group of 41- 50, 51- 60 and $61-70$ yrs.

Segregation of data done according to category. In category IV age group of 11-20 had none case, 21- 30 age group $2.6 \%$ (2 cases) out of which 1.3 $\%$ (1 case) male and $1.3 \%$ (1 case) female, 31-40 age group $2.6 \%$ ( 2 cases) out of which $1.3 \%$ (1 case) male and $1.3 \%$ (1 case) female, $41-50$ age group $5.3 \%$ (4 cases) out of which male $1.3 \%$ (1 case) and $4 \%$ (3 cases) female, 51- 60 age group $6.7 \%$ ( 5 cases) out of which male $1.3 \%$ (1 case) and none female found, 61 -70 age group no case found.

Sensitivity report of Rifampicin data segregation done according to the age groups showed, age group of 11-20 had $6.7 \%$ (5 cases) cases out of which $2.7 \%$ ( 2 case) male and $4 \%$ ( 3 cases) female, 21- 30 age group $23 \%$ (17 cases) out of which $17.6 \%$ (13 cases) male and $5.4 \%$ (4 cases) female, 31-40 age group 30\% (22 cases) male $25.7 \%$ (19 cases) female $4 \%$ (3 cases), $41-50$ age group $21.6 \%$ (16 cases) out of which male $16.2 \%$ (12 cases) and $5.4 \%$ ( 4 cases) female, 51 60 age group $10.8 \%$ ( 8 cases) out of which male $9.4 \%$ ( 7 cases) and $1.3 \%$ ( 1 case) female found, $61-70$ age group $8 \%$ (6 cases) out of which male $5.4 \%$ ( 4 cases) and female $2.7 \%$ ( 2 cases).

Sensitivity report of Isoniazid and Rifampicin data segregation done according to the age groups showed, age group of 11-20 had $4 \%$ (3 cases) cases out of which no male found and $4 \%$ ( 3 cases) female, 21 - 30 age group $13.5 \%$ (10 cases) out of which $8.1 \%$ (6 cases) male and $5.4 \%$ (4 cases) female, 31-40 age group $14.8 \%$ (11 cases) male $10.8 \%$ ( 8 cases) female $4 \%$ (3 cases), 41 50 age group $9.4 \%$ ( 7 cases) out of which male $8.1 \%$ (6 cases) and $1.3 \%$ ( 1 case) female, 51- 60 age group $4 \%$ ( 3 cases) out of which male $4 \%$ (3 cases) and no female found, $61-70$ age group 2.7 $\%$ ( 2 cases) out of which male $1.3 \%$ ( 1 case) and female $1.3 \%$ ( 1 case).

Data reports of PCR which confirmed that $100 \%$ cases were positive for tuberculosis infection. Out of 74 cases $28 \%$ were in category I, $57 \%$ cases in category II, $3 \%$ cases in category in III, $12 \%$ cases in category IV. Three (4\%) cases were presented to center as extrapulmonory tuberculosis. Culture and sensitivity report showed $100 \%$ cases were resistant to Rifampicin, $50 \%$ cases were resistant to isoniazid and $50 \%$ cases were resistant to both.

Immunocompromised status was taken in account and only 1 patient found HIV positive and was a case of pulmonary tuberculosis. 


\section{Discussion}

In the present study, PCR for TB showed the best diagnostic yield followed by acid-fast bacilli staining and culture. Rapid diagnosis of TB is a challenge, especially due to the paucibacillary nature of specimens; inadequate samples, apportioning of samples for various tests (histology/cytology, biochemical analysis, microbiology and PCR) resulting in non-uniform distribution of microorganism ${ }^{8}$. Prompt and accurate diagnosis of tuberculosis is still a dilemma in developing countries where LJ culture is still used as the gold standard for its diagnosis. The culture is time consuming (6-8 weeks) therefore, evidences like histology/cytology along with clinical evaluation are still being used to treat the patient with a full course of anti-tubercular treatment ${ }^{9}$. A wide range of smear positivity between (0-75\%) has been reported in earlier studies $^{10}$. In the present study A total of 74 cases were taken in this study, out of which $73 \%$ (54 cases) of were male and $27 \%$ (20 cases) were female. In this study $86 \%$ (64 cases) cases were sputum positive, out of which $76.6 \%$ (49 cases) were male and $23.4 \%$ (15 cases) female as compare to Stella Sala Soares Lima study which shows $54.2 \%$. Three sputum smears for acid-fast bacilli are recommended for proper diagnosis in pulmonary suspects of $\mathrm{TB}^{\mathbf{1 1}}$ however, WHO has proposed two smears for the diagnosis of TB in countries having functional external quality assurance $^{\mathbf{1 2}}$. Culture using LJ medium has been the gold standard for the diagnosis of tuberculosis for many years in the developing countries. In the present study, the yield of culture positivity was moderately low when compared with the smear positivity. In present study $72.9 \%$ (56) samples were positive on culture, out of which male were $76 \%$ (41 cases) and female $24 \%$ (13 cases). Another study showed a culture sensitivity of 80$85 \%{ }^{13}{ }^{14}$. Detection of Mycobacterium tuberculosis using culture on LJ medium is very specific and detects as few as 10 bacteria per milliliter of specimen as compared to smear that requires about 5000 to 10000 acid-fast bacilli /ml of specimen 13. A quicker and yet accurate diagnosis of Mycobacterium tuberculosis is pivotal in the management of TB. All samples (100\%) were positive for PCR, out of which 75.7 $\%$ (56 cases) were male and $24.3 \%$ (18 cases) were female. The sensitivity of PCR was remarkably high when compared to smear and culture in this study and PCR took much shorter time (1-3 days) as compared to culture (6-8 weeks). The drawbacks of PCR are its high cost, specific requirement of infrastructure and equipment and expertise. The sensitivity of PCR to pick tuberculosis bacteria was $10 \mathrm{fg}$, which is equal to only two bacilli of Mycobacterium tuberculosis ${ }^{15}$ indicating that PCR can pick even a very small amount of bacteria. Another advantage of PCR is that it can also detect nonviable bacilli of cases that are already taking anti tuberculosis treatment or in formalin fixed specimens ${ }^{\mathbf{1 5}}$. At present, it can be concluded that the smear is cheap and rapid method of detecting mycobacterium tuberculosis but it has a very low sensitivity. Culture is more sensitive but it takes a longer time to give results while PCR is specific, rapid, more sensitive but expensive technique, and it should only be used in difficult cases where diagnosis become a challenge.

\section{Bibliography}

1. WHO: Global tuberculosis control surveillance, planning, financing. WHO Report 2006 2006:362

2. Morre DF, Curry JI. Detection and identification of mycobacterium tuberculosis directly from sputum specimen by Amplicore PCR. J Clin Microbiol 1995; 33:2686-91.

3. Truffot-Pernot C, Véziris N, Sougakoff W. Modern diagnosis of tuberculosis. Presse Med 2006; 35 (Pt 2):1739-46.

4. Daniel, T. M. 1990. The rapid diagnosis of tuberculosis: a selective review. J. Lab. Clin. Med. 116:277-282.

5. Pervez MAK, Hasan QN, Rumi MAK, Ahmed S, Salimullah M, Tahera Y et al. PCR can help early diagnosis of pulmonary tuberculosis. 
Southeast Asian JTrop Med Public Health 2003; 34: 147-53.

6. Tiwari V, Jain A, Verma RK. Application of enzyme amplified mycobacterial DNA detection in the diagnosis of pulmonary and extra-pulmonary tuberculosis. Indian $\mathrm{J}$ Med Res 2003; 118: 224-8.

7. Cegielski JP, Devlin BH, Morris AJ. Comparison of PCR, culture, and histopathology for diagnosis of tuberculous Pericarditis. J Clin Microbiol 1997;35: 3254-7.

8. Kesarwani RC, Pandey A, Misra A.. Polymerase chain reaction (PCR): its comparison with conventional techniques for diagnosis of extra-pulmonary tubercular disease. Indian J Surgery 2004; 66: 84-8.

9. World Health Organization. Treatment of tuberculosis: guidelines for national programmes. [Geneva]: WHO;1993.

10. Kamboj SS, Goel MM, Tandon P.l. Correlation study of histopathology and bacteriology in patients of tubercular lymphadenitis. Indian J Chest Dis Allied Sci 1994; 36:187-91.

11. Saleem S, Shabbir I, Iqbal I. Value of three sputum smear microscopy in diagnosis of pulmonary tuberculosis. Pak J Med Res 2007; 46: 94-7.

12. World Health Organization. Proposed reduction of number of smears for the diagnosis of pulmonary TB: ackground document. Available from: URL:http://www.who.int/tb/dots/laboratory/R eduction $\% 20$ of $\% 20$ smears.

13. Dunlap NE, Bass J, Fujiwara P. Diagnostic standards and classification of tuberculosis in adults and children. Am J Respir Crit Care Med 2000; 161: 1376-95.

14. Yeager IIJ Jr, Lacy J, Smith L. Quantitative studies of mycobacterial populations in sputum and saliva. Am Rev Respire Dis 1967; 95: 998-1004.
15. Negi SS, Anand R, Basir SF. Protein antigen $b$ (Pab) based PCR test in diagnosis of pulmonary and extra pulmonary tuberculosis. Indian J Med Res 2006; 124: 81-8. 